\title{
Impacto del consumo episódico excesivo de alcohol en la adolescencia. ¿Lo estamos haciendo bien?
} Impact of Binge Drinking (BD) in
Adolescence. Are we doing it right?

\author{
ana Magdalena Vargas-Martínez*, Marta Trapero-Bertran**, Eugenia Gil-García***, \\ Marta Lima-SERrano***. \\ * Facultad de Ciencias de la Salud. Universidad Pontificia de Salamanca. Salamanca, España. ** Facultad de Ciencias \\ Económicas y Sociales. Universitat Internacional de Catalunya (UIC). Barcelona, España. *** Departamento de Enfermería. \\ Facultad de Enfermería, Fisioterapia y Podología. Universidad de Sevilla. Sevilla, España.
}

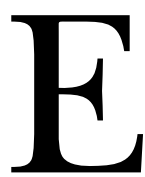

stimado Sr. Editor,

El consumo excesivo de alcohol y los trastornos de uso de alcohol son la causa principal de muerte y discapacidad a nivel mundial (OMS, 2014). Según la Organización Mundial de la Salud, el 10\% de las muertes entre adolescentes (con edades entre los 1519 años) en la Región Europea fueron atribuibles a alcohol (Drost et al. 2016).

Hoy en día, el consumo episódico excesivo de alcohol (BD, binge drinking) es uno de los patrones de consumo de alcohol más prevalentes. En el año 2015, se estimó que el $35 \%$ de los adolescentes europeos con edades entre los 1516 años participó al menos una vez en BD en los últimos 30 días (The ESPAD Group, 2016). Es más, en España, según la serie de encuestas sobre el uso de drogas en enseñanzas secundarias, ESTUDES 2014-2015, el 32,2\% han participado en BD al menos una vez en el último mes (Plan Nacional de Drogas, 2016), mientras que un estudio reciente de Golpe, Gómez, Braña, Varela y Rial (2017) llegó a la conclusión que el $33,1 \%$ de los adolescentes españoles consumieron alcohol de manera intensiva en el último año, y el $20 \%$ en el último mes (3 o más bebidas alcohólicas por ocasión de consumo y embriaguez). Además, el 19,8\% de los adolescentes tenían un consumo de alcohol de riesgo, sin diferencias significativas por género. Romo-Avilés, Marcos-Marcos, Tarragona-Camacho, Gil-García y Marquina-Márquez (2016) encontraron diferencias menores entre niños y niñas respecto de la cantidad de alcohol consu- mida durante la participación en un botellón. Esto sugiere que ha aumentado el consumo intensivo de alcohol y el BD en las niñas.

En la Unión Europea, los costes atribuidos al alcohol se estimaron en $€ 125$ billones en el año 2003. En España, el coste social total del consumo de alcohol puede representar el 1\% del producto interior bruto (más de $€ 10$ billones) (Pulido, Indave-Ruiz, Ruiz-García, Bartroli y Barrio, 2014).

No hemos encontrado ningún estudio sobre los costes asociados con el BD y el consumo de alcohol entre menores en Europa, pero estudios anteriores han mostrado que consumidores jóvenes de alcohol tienen mayor riesgo de: ser víctimas y perpetradores de violencia juvenil; tener un nivel bajo de estudios; tener menor expectativa de realizar estudios universitarios; y suponer una carga en términos económicos para el sistema de justicia penal y para el sector de la educación (OMS, 2014).

Basado en la evidencia, BD se relaciona principalmente con efectos agudos en jóvenes, como intoxicaciones agudas, lesiones accidentales e intencionadas, accidentes de tráfico, problemas académicos debido a la disminución del rendimiento cognitivo y la alteración cerebral como del absentismo escolar debido a los síntomas de la resaca que resulta de la intoxicación aguda de alcohol, relaciones sexuales sin protección y no planificadas, consumo de otras drogas, problemas con la ley debido a la reducción de las capacidades cognitivas y verbales para resolver conflictos y el desarrollo de un trastorno de uso de alcohol en

Recibido: Octubre 2017; Aceptado: Noviembre 2017.

Enviar correspondencia a:

Ana Magdalena Vargas-Martínez. Facultad de Ciencias de la Salud. Universidad Pontificia de Salamanca. Calle Compañía 5. Salamanca 37002 (España). Teléfono móvil: 670709328. E-mail: vargasmartinezam@gmail.com 
la adultez (Pulido et al., 2014, Windle y Windle, 2017). Las investigaciones de Windle y Windle en el año 2017 encontraron que la fiabilidad diagnóstica de problemas relacionados con el alcohol entre adolescentes para predecir la dependencia del alcohol 7 años más tarde fue del 74\%. En España, la prevalencia anual de intoxicación etílica aguda autoinformado superó el $30 \%$ de la población con edades entre los 15-34 años en el año 2011, siendo más elevada en los adolescentes con edades entre los 15-16 años (Pulido et al., 2014).

No obstante, todos estos eventos adversos o agudos podrían estar asociados con consecuencias económicas en términos de, por ejemplo, asistencia sanitaria ambulatoria, ingresos hospitalarios, algunos costes directos asociados con la violencia, tales como cuidados de emergencia, servicios policiales o de justicia penal, accidentes de tráfico que conllevan atención sanitaria y absentismo escolar (Pulido et al., 2014; OMS, 2014).

Dada la prevalencia elevada y las consecuencias sanitarias, sociales y económicas del uso de alcohol y de $\mathrm{BD}$, la necesidad de implementar estrategias preventivas es obvia. ¿Qué medidas para la prevención del uso de alcohol se implementan en la actualidad? Hasta la fecha, se han diseñados programas diferentes para prevenir el uso de alcohol entre adolescentes (Foxcroft y Tsertsvadze, 2012; Jander, Crutzen, Mercken, Candel y de Vries, 2016). Hoy en día hay algunos programas de prevención, informáticos y online, pero su uso es habitualmente más extendido a nivel internacional.

Un metaanálisis de intervenciones a medida basadas en la web para cambiar conductas relacionadas con la salud mostró que dichas intervenciones tienen un impacto significativo en las tasas de factores de riesgo conductuales (Krebs, Prochaska y Rossi, 2010). Primero, estas intervenciones tienen el potencial de alcanzar a muchas personas de clases sociales y edades diferentes. Esto es posible porque un porcentaje importante de la población actualmente tiene acceso a Internet. Segundo, la información personalizada es percibida como más relevante que la información no personalizada (Schulz et al., 2014).

A pesar de la existencia de intervenciones a niveles internacional y nacional (Foxcroft y Tsertsvadze, 2012) con el fin de prevenir el uso de alcohol, su eficiencia y relación coste-eficacia ha sido evaluada en muy pocas ocasiones (Drost et al., 2016). Dada la coyuntura actual de presupuestos limitados, analizar la eficacia en términos de coste de las intervenciones es necesaria para la toma de decisiones en cuestiones relacionadas con la salud. Por lo tanto, el análisis de la relación coste-eficacia es una herramienta útil para valorar si una intervención es interesante, y para influenciar en las políticas y la planificación sanitaria.

En definitiva, existen huecos importantes respecto de la información sobre los impactos negativos resultantes del consumo de alcohol en España, en particular en la esti- mación de su carga sobre la mortandad y la morbilidad, la prevalencia de los trastornos de uso de alcohol, los costes sociales del consumo y la eficiencia de las intervenciones o los programas preventivos. Por lo tanto, es necesario evaluar la carga económica y valorar las intervenciones en BD en términos económicos.

\section{Contribuciones de los autores}

AM Vargas-Martínez preparó el borrador inicial y coordinó el trabajo. E Gil-García revisó el trabajo y aportó la perspectiva de género. M Lima-Serrano y M Trapero-Bertran hicieron contribuciones sustanciales y revisiones importantes al contenido del texto. El conjunto de autores aprobó la versión definitiva del mismo.

\section{Financiación}

Este estudio ha sido preparado en el marco del proyecto de investigación "ALCOHOL ALERT: Design, validation and evaluation of the program of selective prevention of alcohol abuse in adolescents: web-based computer-tailored intervention" (PI-0031-2014) [ALERTA DE ALCOHOL: Diseño, validación y evaluación del programa de prevención selectivo del abuso de alcohol entre adolescentes: intervención informático online], financiado por la Fundación Pública de Andalucía "Progreso y Salud" para la financiación de la investigación y la innovación biomédica en las ciencias de la salud en Andalucía para el año 2014 (Terapias Avanzadas).

\section{Conflicto de intereses}

Ninguna.

\section{Referencias}

Drost, R. M., Paulus, A. T. G., Jander, A. F., Mercken, L., de Vries, H., Ruwaard, D., y Evers, S. M. (2016). A web-based computer-tailored alcohol prevention program for adolescents: Cost-Effectiveness and intersectoral costs and benefits. Journal of Medical Internet Research, 18, e93. doi:10.2196/jmir.5223.

Foxcroft, D. R., y Tsertsvadze, A. (2012). Universal school-based prevention programs for alcohol misuse in young people. Evidence-Based Child Health, 7, 450-575. doi:10.1002/ebch.1829.

Golpe, S., Gómez, P., Braña, T., Varela, J., y Rial, A. (2017). The relationship between consumption of alcohol and other drugs and problematic Internet use among adolescents. Adicciones, 29, 268-277. doi:10.20882/adicciones.959.

Jander, A., Crutzen, R., Mercken, L., Candel, M., \& de Vries, H. (2016). Effects of a Web-Based Computer-Tailo- 
red Game to Reduce Binge Drinking Among Dutch Adolescents : A Cluster Randomized Controlled Trial. Journal of Medical Internet Research, 18, e29. doi:10.2196/jmir.4708.

Krebs, P., Prochaska, J.O. y Rossi, J.R. (2010). A meta-analysis of computer-tailored interventions for health behaviour change. Preventive Medicine, 51: 214-221.

Organización Mundial de la Salud (OMS). (2014). Global status report on alcohol and health. Extraído de http:// www.who.int/substance_abuse/publications/global_alcohol_report/en/.

Plan Nacional sobre Drogas. (2016). Encuesta sobre el uso de drogas en enseñanzas secundarias en España (ESTUDES) 2014-2015. Madrid, España: Delegación del Gobierno para el Plan Nacional sobre Drogas, Ministerio de Sanidad, Servicios Sociales e Igualdad. Extraído de http://www.pnsd.msssi.gob.es/profesionales/sistemasInformacion/sistemaInformacion/pdf/2016_ESTUDES_2014-2015.pdf.

Pulido, J., Indave-Ruiz, B.I., Ruiz-García, M., Bartroli, M. \& Barrio, G. (2014). Estudios poblacionales en España sobre daños relacionados con el consumo de alcohol. Revista de Salud Pública, 8: 493-513.

Romo-Avilés, N., Marcos-Marcos, J, Tarragona-Camacho, A., Gil-García, E. y Marquina-Márquez, A. (2016). "I like to be different from how I normally am": Heavy alcohol consumption among female Spanish adolescents and the unsettling of traditional gender norms. Drugs: Education, Prevention and Policy. Avance de publicación online. doi:10.1080/09687637.2016.1259390.

Schulz, D.N., Smit, E.S., Stanczyk, N.E., Kremers, S.P., de Vries, H. y Evers, S.M. (2014). Economic evaluation of a web-based tailored lifestyle intervention for adults: findings regarding cost-effectiveness and cost-utility from a randomized controlled trial. Journal of Medical Internet Research, 16, e91. doi:10.2196/jmir.3159.

The ESPAD Group. (2016). ESPAD Report 2015: Results from the European School Survey Project on Alcohol and Other Drugs. Lisbon: European Monitoring Centre for Drugs and Drug Addiction (EMCDDA). Retrieved at http://www.espad.org/sites/espad.org/files/ESPAD_report_2015.pdf.

Windle, M. y Windle, R. (2017). The Measurement of Adolescent Alcohol Problems via Item Response Theory and Their 15-Year Prospective Associations with Alcohol and Other Psychiatric Disorders. Alcoholism: Clinical and Experimental Research, 41, 399-406. doi:10.1111/acer.13301. 